\title{
Post-natal cardiomyocytes can generate iPS cells with an enhanced capacity toward cardiomyogenic re-differentation
}

\author{
R Rizzi ${ }^{1,2}$, E Di Pasquale ${ }^{2,3}$, P Portararo ${ }^{2}$ R Papait ${ }^{2,3}$, P Cattaneo ${ }^{2,3}$, MVG Latronico ${ }^{2}$, C Altomare ${ }^{4}$, L Sala ${ }^{4}$, A Zaza ${ }^{4}$, E Hirsch ${ }^{5}$, \\ L Naldini ${ }^{6}$, G Condorellii, ${ }^{*, 7}$ and C Bearzi ${ }^{1,2}$
}

Adult mammalian cells can be reprogrammed to a pluripotent state by forcing the expression of a few embryonic transcription factors. The resulting induced pluripotent stem (iPS) cells can differentiate into cells of all three germ layers. It is well known that post-natal cardiomyocytes (CMs) lack the capacity to proliferate. Here, we report that neonatal CMs can be reprogrammed to generate iPS cells that express embryonic-specific markers and feature gene-expression profiles similar to those of mouse embryonic stem (mES) cell and cardiac fibroblast (CF)-derived iPS cell populations. CM-derived iPS cells are able to generate chimeric mice and, moreover, re-differentiate toward CMs more efficiently then either CF-derived iPS cells or mES cells. The increased differentiation capacity is possibly related to CM-derived iPS cells retaining an epigenetic memory of the phenotype of their founder cell. CM-derived iPS cells may thus lead to new information on differentiation processes underlying cardiac differentiation and proliferation.

Cell Death and Differentiation (2012) 19, 1162-1174; doi:10.1038/cdd.2011.205; published online 20 January 2012

Cell-based therapies constitute a promising therapeutic option for many pathologies. Unfortunately, the development of this approach for improving cardiac function in heart failure is hindered by the difficulty in obtaining a reliable source of functional cardiomyocytes (CMs). In fact, adult CMs are characterized by a very low proliferation rate, and this limits their use for replacement therapy. ${ }^{1}$ As a consequence, many cell types have been tested for their potential as a source of $\mathrm{CMs}^{2}{ }^{2}$ These have included mesenchymal and hematopoietic stem cells, skeletal myoblasts, endothelial progenitors, and resident cardiac stem cells. ${ }^{3}$ However, the use of these cells as $\mathrm{CM}$ progenitors is controversial.

To date, embryonic stem (ES) cells constitute the only reliable source of functional CMs. ES cells derive from the blastocyst or early epiblast; when cultured in vitro, they mature into so-called embryoid bodies (EBs), teratoma-like structures that contain cells able to generate the three embryonic layers (ectoderm, endoderm, and mesoderm). ${ }^{4}$ Human ES cells can generate functional CMs with neonatal cell characteristics. ${ }^{5}$ However, the use of human ES cells is hampered by difficulties in lineage generation from in vitro fertilized cells, their immunogenicity - which is, nevertheless, lower compared with that of adult cells - and above all by restrictions in some countries on the generation of new embryonic cell lines. ${ }^{6}$

Induced pluripotent stem (iPS) cells - genetically modified cells generated by the integration of embryonic transcription factor genes (Oct4, Sox2, KIf4, and c-Myc (OSKM)) into the genome of adult somatic cells - may overcome some of the above-mentioned difficulties related to the use of human ES cells. ${ }^{7,8}$ iPS cells have been derived from various sources, such as gastric epithelial cells, hepatocytes, embryonic fibroblasts, adult tail-tip fibroblasts, pancreatic cells, B lymphocytes, and neural stem cells from mouse, as well as keratinocytes, skin fibroblasts, and peripheral blood cells from humans. ${ }^{9}$ These cells form germ-line adult chimeras and possess self-renewal and pluripotency characteristics that are similar to ES cells.

In addition to their potential in cell-based therapies, iPS cells may be used in the future for drug development and testing in vitro, decreasing the necessity of in vivo toxicology studies. iPS cells have already been generated from cells of

${ }^{1}$ Cell Biology and Neurobiology Institute, National Research Council of Italy (CNR), Roma, Italy; ${ }^{2}$ Istituto Ricovero Cura Carattere Scientifico MultiMedica, Milan, Italy; ${ }^{3}$ Institute of Genetic and Biomedical Research, National Research Council of Italy (CNR), Milan, Italy; ${ }^{4}$ Department of Biotechnologies and Biosciences, University of Milano-Bicocca, Milan, Italy; ${ }^{5}$ Department of Genetics, Biology and Biochemistry and Molecular Biotechnology Center, University of Torino, Torino, Italy; ${ }^{6}$ Fondazione San Raffaele Monte Tabor, Milan, Italy; ${ }^{7}$ Department of Medicine, University of California San Diego, La Jolla, CA, USA and ${ }^{8}$ Department of Medicine, CNR, Milan, Italy *Corresponding author: G Condorelli, Department of Medicine, University of California San Diego, La Jolla, CA 92093-0613C, USA. Tel: + 1858 822 5563;

Fax: +1 858534 3027; E-mail: gcondorelli@ucsd.edu

Keywords: iPS cells; cardiomyocytes; cardiac fibroblasts; cardiac repair

Abbreviations: Ach, acetylcholine; Aldh3a1, aldehyde dehydrogenase family 3, subfamily A; ALP, alkaline phosphatase; BMP2, bone morphogenic protein 2; C1QTNF2, C1q tumor necrosis factor related protein 2; CFs, cardiac fibroblasts; CM, cardiomyocyte; DEG, differentially expressed genes; EBs, embryoid bodies; EC, endothelial cell; ES, embryonic stem; GFP, green fluorescent protein; GO, Gene ontology; Igfbp5, insulin-like growth factor binding protein 5; IP3, inositol trisphosphate; iPS, induced pluripotent stem; Lrp6, low-density lipoprotein receptor-related protein 6; mES, mouse embryonic stem; MHC, myosin heavy chain; MLC, myosin light chain; nAChR, nicotinic acetylcholine receptor; Oct4, octamer-binding transcription factor 4; OSKM, Oct4, Sox2, Klf4, and c-Myc; qRT-PCR, quantitative Real Time PCR; RyR, ryanodine receptor; SMC, smooth muscle cell; SSEA-1, stage-specific embryonic antigen 1; TAZ, transcriptional co-activator with PDZ-binding motif; TBX5, T-boX transcription factor 5; TGF- $\beta$, transforming growth factor beta; TNNI3, cardiac troponin-I; Wnt7a, wingless-related MMTV integration site 7; $\alpha$-SARC, alpha-sarcomeric actin.

Received 26.5.11; revised 30.11.11; accepted 13.12.11; Edited by RA Knight; published online 20.1.12 
patients with degenerative pathologies, ${ }^{10}$ opening up the possibility of using patient-specific iPS cells to generate study models of disease.

In the present study, we determined the ability of post-natal CMs and cardiac fibroblasts (CFs) to act as a source of iPS cells. Our results indicate that the genome of differentiated cardiac cells can be reset to a pluripotent state. Moreover, we found a genetic propensity in CM-derived iPS cells to re-differentiate toward the fate of their founder cell.

\section{Results}

Primary culture of CMs and CFs and generation of iPS cells with OSK factors. In an attempt to generate iPS cells from CMs, we started by transducing primary CM cultures with lentiviral vectors expressing four pluripotency factors (OSKM) along with green fluorescent protein (GFP) as a tracker. We found that $\mathrm{CMs}$ were capable of generating clones with this procedure. However, the number of clones produced was significantly less than that generated by CFs, even though the transduction index was high in both cell types (Supplementary Figure 1). We reasoned that this low clonogenic efficiency could be due to the large expression cassettes of the OSKM lentiviral vectors used, which contain genes for an embryonic factor plus the GFP marker, limiting the efficiency of transduction and, consequently, of the reprogramming process. To overcome this problem, we decided to transduce cells with vectors harboring bicistronic constructs encoding Oct4 and either Sox2 or KIf4, without the GFP gene (OSK lentiviral vectors) (Figure 1a).

In addition, we decided to improve another limit of our initial procedure, that is, the purity of the CM population employed. Even if the percentage of CF contamination was very low with the culture methods used, we could not assume that the clones derived from CM cultures were all generated from CMs. Thus, in order to eliminate the chances of generating iPS cells from contaminating CFs, we adopted a method previously employed for monitoring the differentiation of mouse ES (mES) cells into CMs: ${ }^{11} 4$ days before transduction with OSK lentiviral vectors, primary cultures were incubated with a cardiac-specific vector in which GFP expression is driven by a short fragment of the cardiac troponin-I (TNNI3) proximal promoter linked to a cardiac $\alpha$-actin enhancer (TNNI-GFP lentivirus vector). The brightest GFP-positive cells were sorted and plated directly onto gelatin-coated dishes, ready for incubation with OSK lentiviral vectors (Supplementary Figure 2).

FACS analysis revealed that $\approx 60 \%$ of cells originating from $\mathrm{CM}$ cultures and only $\approx 0.9 \%$ of cells originating from CF cultures expressed GFP after transduction with the TNNI3-GFP lentivirus vector (Figure 1b, upper left panel; Supplementary Figure 3 and related Supplementary Movie S1). All these GFP-positive cells expressed $\alpha$-sarcomeric actin $(\alpha-S A R C)$, indicating that they were CMs (Figure $1 b$, upper right panel). The degree of purity of the sorted brightest cells was confirmed by FACS and by quantitative Real Time PCR (qRT-PCR) analyses of CM- and CF-specific markers (Figures 1b, lower panels, and 1c).
Moreover, to exclude the possibility that progenitor cells or immature $\mathrm{CMs}$ were contributing to any reprogramming taking place, we tested for the expression of antigens and genes that characterize developing cardiac cells. A contribution by putative cardiac progenitors was ruled out by the absence of c-kit-, isl1-, and Sca1-positivity in cells selected for TNNI3driven GFP expression (Figure 1d). In addition, the expression of T-box transcription factor 5 (TBX5), beta-myosin heavy chain $(\beta-M H C)$, and $N K X 2.5$ - genes expressed in immature CMs - was either greatly reduced or zero in cells after the sorting step (Figure 1e). In contrast, genes considered late, such as alpha-myosin heavy chain $(\alpha-M H C)$ and $T N N I$, were highly expressed after selection. As expected, GATA4 was always expressed, and mES cells never expressed CMrelated genes.

We then used OSK lentiviral vectors to induce reprogramming of cardiac cells. Compared with transduction with OSKM constructs, reprogramming was significantly increased in both compartments with the use of only OSK. Indeed, we counted the number of colonies/plate expressing alkaline phosphatase (ALP) - a hydrolase typical of the undifferentiated mES cell state - 18 days after incubation with OSK lentiviral vectors and found that the $\mathrm{CM}$ compartment became significantly more prone to reprogramming than the CF one (Figure 2a; Supplementary Figure 4). Moreover, 5-bromo-2'-deoxyuridine incorporation experiments conducted on GFP-expressing CMs revealed that DNA synthesis increased after transduction with OSK (Figure $2 b$ ). These results strongly suggest that neonatal CMs transduced with the three transcription genes proliferate while de-differentiating. Visual confirmation attested that the clones from the CM compartment spread much faster and become bigger than those generated from CFs. With increased number of culture passages, the morphology of CM- and CF-derived iPS cells increasingly resembled that of ES cells (Figure 2c).

Molecular characterization of CM- and CF-derived iPS cells. Immunofluorescence, colorimetric assay of ALP, and molecular analyses were conducted at different time points after OSK transduction. Twelve days after incubation with OSK lentiviral vectors, we found that cells of $\mathrm{CM}$ - and CF-derived colonies expressed stage-specific embryonic antigen 1 (SSEA-1), an embryonic surface marker (Figure 2d). In addition, cells were positive for ALP, and endogenous octamer-binding transcription factor 4 (Oct4) was found reactivated. These results further proved that reprogramming was taking place.

Teratoma formation. We confirmed the pluripotency of newly formed CM-derived iPS cells by the ability to form teratomas when injected subcutaneously in NOD-scid mice. Tumors were found in all these mice after 4 weeks. Hematoxylin/eosin staining demonstrated the presence of the three germ cell layers (Figure 2e). Moreover, the karyotype did not present abnormalities and chromosome conformation was considered normal (Figure 2f).

Generation of chimeras. We next investigated whether CM-derived iPS cells were of a sufficient quality to produce adult chimeras. Injection of CM-derived iPS cells generated 
a

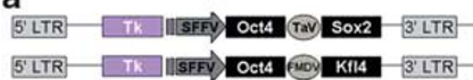

b CM Enrichment
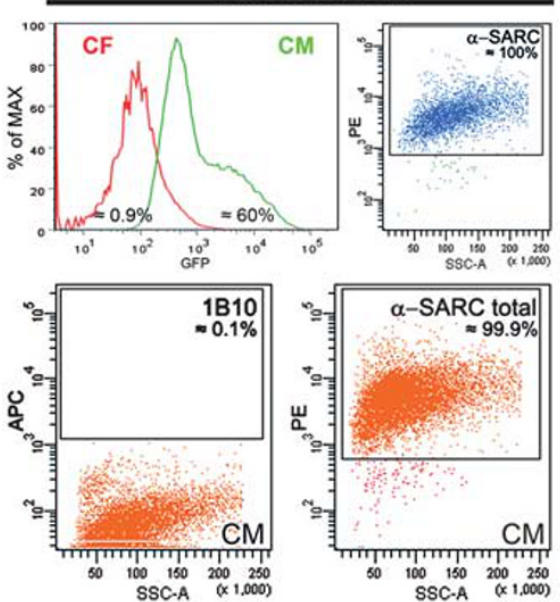

C

CM Purification

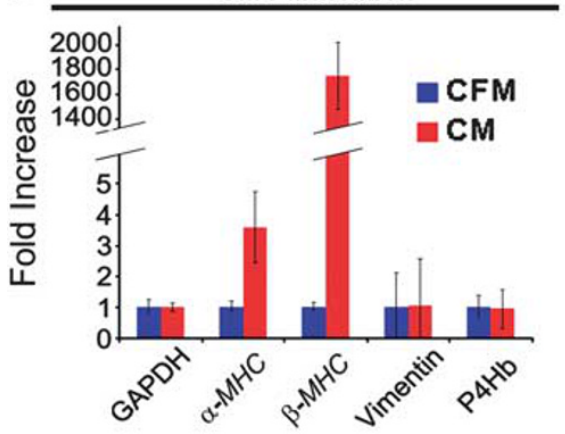

d

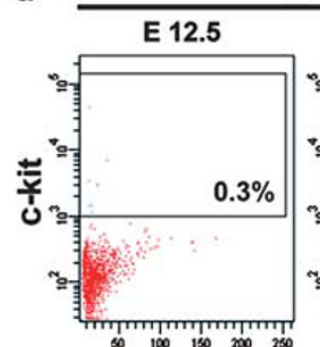

Cardiac Progenitor Markers After Purification
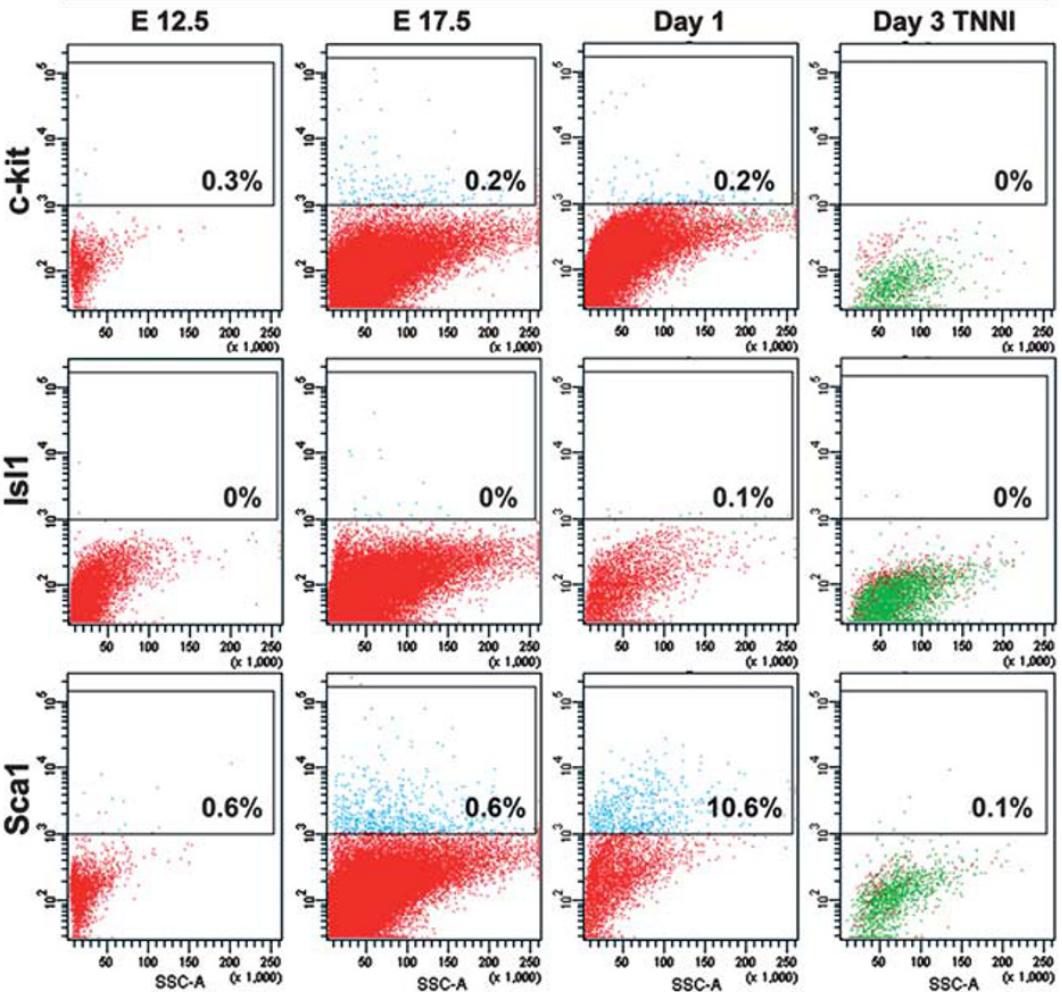

e

Cardiac Gene Expression After Purification
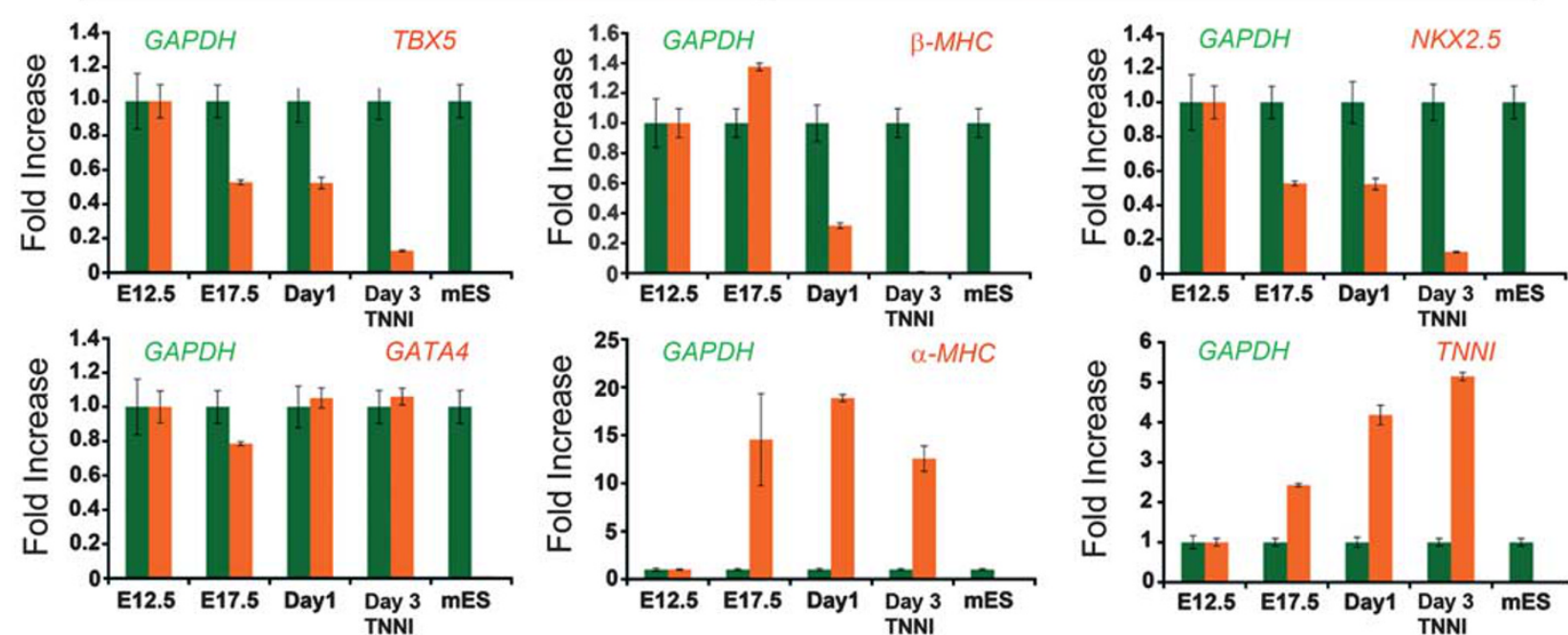

Figure $1 \mathrm{CM}$ - and CF-derived iPS cells obtained through transduction with OSK factors. (a) Maps of the two bicistronic OSK constructs used to improve the efficiency of clone generation in CMs. LTR, long terminal repeats; Tk, thymidine kinase; SFFV, spleen focus-forming virus promoter; TaV, Thosea asigna virus 2A-like sequence; FMDV, foot-andmouth disease virus 2A-like sequence. (b) FACS analysis showing the percentage of GFP-positive cells in cultures of CFs and CMs after incubation with the TNNI3-GFP lentivirus vector (upper left panel); scatter plot of $\alpha$-SARC-expressing GFP-positive cells from a CM culture (upper right panel); FACS analysis on the brightest sorted CMs demonstrating the purity of the population (lower right and left panels). $\alpha$-SARC, alpha-sarcomeric actin; 1B10, fibroblast surface protein marker; PE, phycoerythrin; APC, allophycocyanin. (c) Enrichment of the CM population demonstrated by QRT-PCR. CMs do not express the fibroblast marker prolyl 4-hydroxylase (P4Hb). An axis break has been introduced to remove uninformative areas. CFM, starting cardiac population containing CMs and non-CMs; CM, sorted CM population. (d) Scatter plots of c-kit, Isl1, and Sca1 expression in cardiac cells obtained from hearts at days 12.5 and 17.5 of gestation, 1 day after birth, and after TNNI-sorting. (e) qRT-PCR analysis of control mES cells and cardiac cells obtained from hearts at days 12.5 and 17.5 of gestation, 1 day after birth, and after TNNI sorting 
from CD1 mice (white furred) into blastocysts of C57BL/6 mice (black furred) gave rise to newborn chimeras that developed into adulthood and that showed coat-color chimerism, indicating differentiation of iPS cells into functional melanocytes (Figure 2g).

Expression of OSK factors and cardiac genes. qRT-PCR was conducted to determine the temporal dynamics of gene expression during de-differentiation in $\mathrm{CMs}$ transduced with OSK. Suppression of the exogenous human embryonic genes (Oct4, Sox2, and Klf4) and endogenous mouse cardiac genes ( $T N N I, \alpha-M H C$ ) occurred at around day 30 post-transduction and was concurrent to re-expression of endogenous mouse embryonic genes (Oct4, Nanog) (Figure 2h). The cells had therefore been reprogrammed at around this time and had reached a state of multipotency.

An increase in transcription of exogenous Oct4 and a modest expression of $\alpha-M H C$ was noticed at day 18 of reprogramming. According to a recent study, during the period of de-differentiation there can be temporary expression of exogenous Oct4, which inhibits the transcription of Sox2, permitting subsequent mild activation of differentiation markers. ${ }^{12}$ However, as long as the expression of exogenous Oct4 is transient, the reprogramming process progresses.

Global gene expression. Pearson's correlation of the global gene expression profiles obtained with DNA microarrays revealed that CM- and CF-derived iPS cells are clustered closely with mES cells (Figure 3a). Samples obtained from each stem cell type always had an inter-sample correlation of more than 0.90 (Supplementary Figure 5).

When the threshold was set at a twofold change, 319 genes resulted differentially expressed in CM-derived iPS cells versus mES cells, whereas 381 genes were differentially expressed in CF-derived iPS cells versus mES cells. Out of these differences, only 172 overlapped in the two iPS cell compartments (Figure $3 b$ ). These differences were reduced to 87 genes when the threshold was set at a fivefold change (Figure 3c). At this threshold, genes that were differently upregulated in both iPS cell types versus mES cells included actin-binding protein MIPP and Brachyury. Other genes were upregulated more in CM-derived iPS cells than in CF-derived iPS cells, such as insulin-like growth factor binding protein 5 (Igfbp5), C1q tumor necrosis factor related protein 2 (C1QTNF2), and aldehyde dehydrogenase family 3 , subfamily $A 1$ (Aldh3a1).

Gene ontology (GO) annotation of the iPS cell-sourcedependent differentially expressed genes (DEG) list. We hypothesized that some of the iPS cell-source-dependent DEGs would be involved in molecular pathways important for differentiation in CMs. In order to identify which types of global cellular processes or specific molecular functions were responsible for the propensity of $\mathrm{CM}$-derived iPS cells to become CMs, the DEG list was first annotated using the GO database. Out of the genes found differentially expressed with the twofold-change threshold, 463 genes were assigned to biological process, cellular component, and molecular function categories. The highest percentages of genes in the biological process domain regarded cellular process, biological regulation, and regulation of biological process (Figure 3d, upper pie chart); in the molecular function domain, the highest numbers of genes were part of binding, catalytic activity, and molecular transducer activity (Figure 3d, middle pie chart); in the cellular component domain, cell, cell part, and organelle had the most hits (Figure 3d, lower pie chart). These data confirm that CM- and CF-derived iPS cells are similar, but not identical, to each other and to mES cells.

Expression profile of genes involved in cardiovascular and stemness pathways. The genes found significantly differentially expressed were then grouped in relationship to various pathways involved in cardiac development and stemness using the GeneSifter software (PerkinElmer, Seattle, WA, USA); results were classified according to the terminology given by this software (Supplementary Figure 6). For the smooth muscle cell (SMC) differentiation pathway, we found that although there were no significant differences among the three stem cell types, CM-derived iPS cells tended to cluster with CF-derived iPS cells; in contrast, CM-derived iPS cells clustered with mES cells for genes related to endothelial cell (EC) differentiation (Figure 4, upper-left panels). The genes that we found significantly upregulated in CM-derived iPS cells were wingless-related MMTV integration site 7 (Wnt7a), transcriptional co-activator with PDZ-binding motif (TAZ), and low-density lipoprotein receptor-related protein 6 (Lrp6), which was significantly upregulated in CF-derived iPS cells as well (Figure 4, histograms).

Cardiogenic potential of CM- and CF-derived iPS cells in vitro. Once the de-differentiation process was proven to take place, we sought to determine whether it was possible to differentiate CM- and CF-derived iPS cells into CMs. To this end, we studied mouse embryonic fibroblast (MEF)-derived iPS cells along with mES cells and CM- and CF-derived iPS cells, and exposed EBs grown from these cells to bone morphogenetic protein 2 (BMP2), according to published procedures. ${ }^{13}$ After 3 days, EBs were collected and stained for Flk-1, an embryonic-stage cardiomyogenic-lineage precursor marker. ${ }^{14}$ We found that Flk-1 positivity was present at this time in several clones originating from CM- (Figure 5a, left panel) or CF-derived iPS cells exposed to BMP2. FACS analysis performed at early time points of differentiation showed that CM-derived iPS cells started to express Flk-1 before the other cell types tested (Figure 5b). Positivity for c-kit, which is expressed in putative cardiac progenitor cells, ${ }^{15}$ was not encountered (Figure $5 a$, right panel).

Western blotting was then carried out for cardiac markers to obtain proof of CM differentiation from iPS cells. We found that $\alpha$-SARC, TNNI, myosin light chain $2 \mathrm{~V}$ (MLC $2 \mathrm{~V}$ ), and NKX2.5 were expressed in cells upon exposure to BMP2 (Figure $5 \mathrm{c}$ ). MLC 2A - the atrial MLC isoform - was not expressed, indicating that cells were more frequently differentiating toward the ventricular lineage.

Beating areas started to appear 6 days after BMP2 exposure (Supplementary Movies S2 and S3). The number 

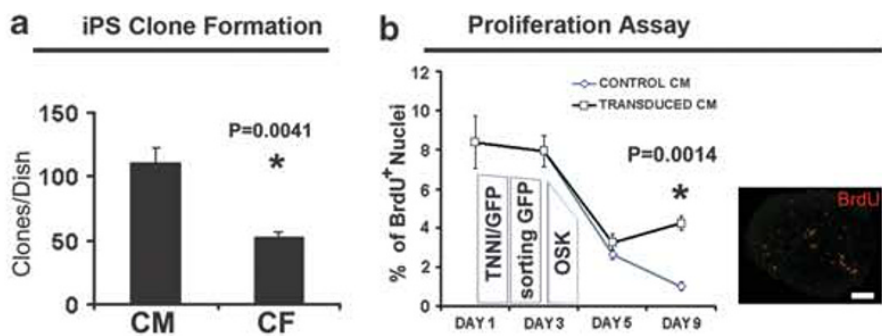

c

Morfphology During Re-Programming Process

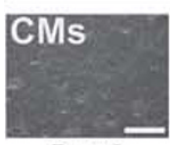

Day 0

Day 3
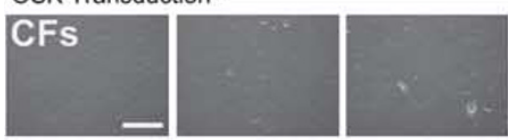

d

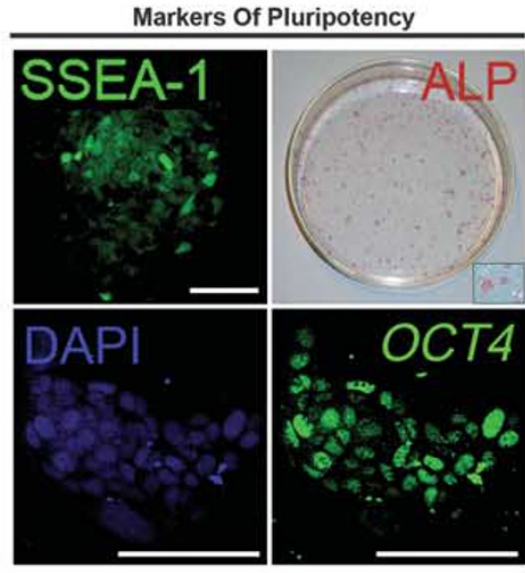

f

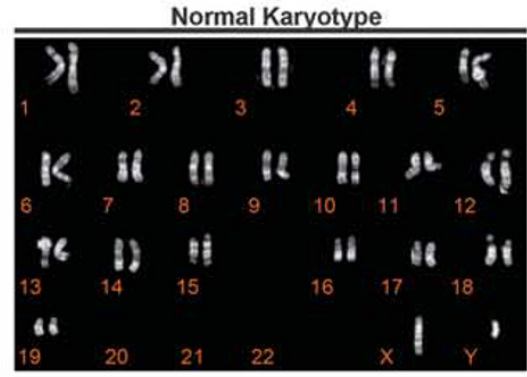

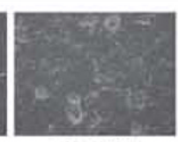

Day 9

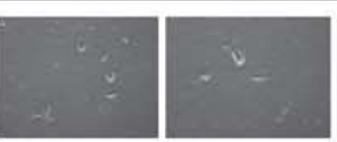

Day 12

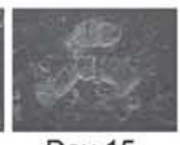

Day 15
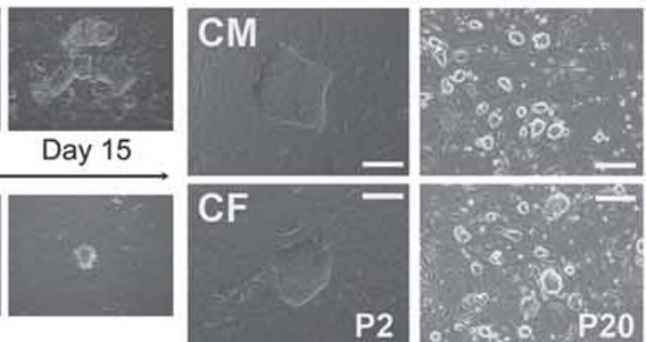

P2

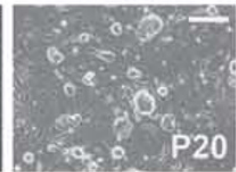

e

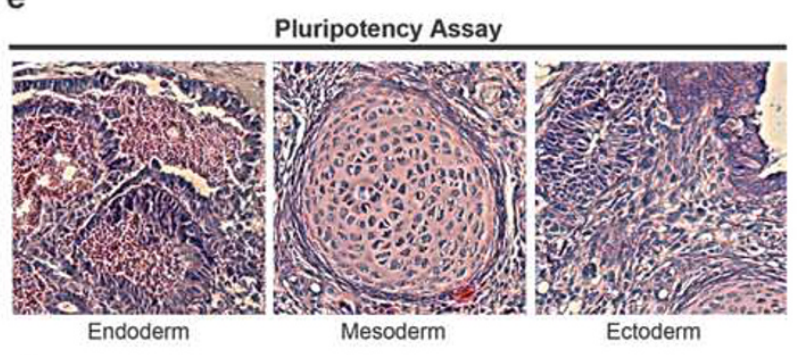

g

Germline Competent Chimera

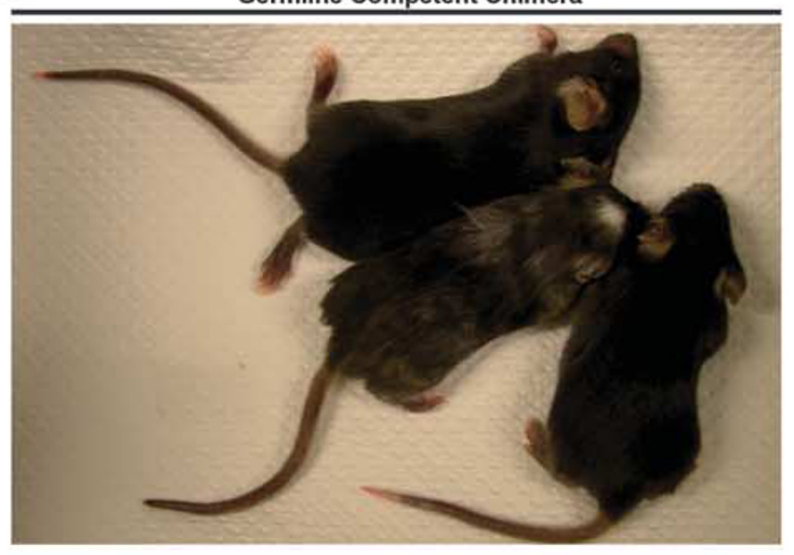

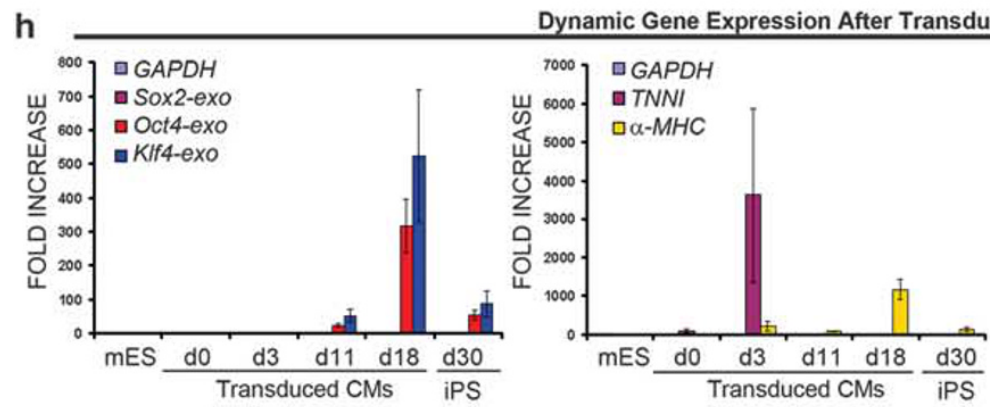

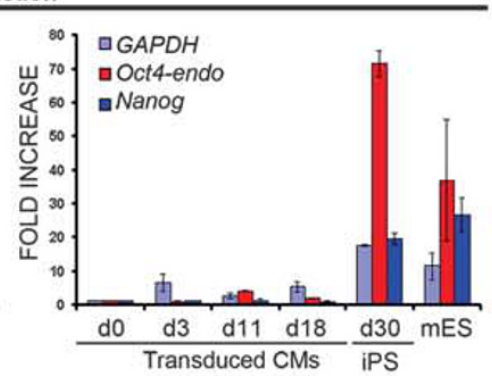


of these beating areas was greater in cultures originating from CM-derived iPS cells (Figure $5 d$ ). The greater propensity of CM-derived iPS cells to become CMs compared with CF- and MEF-derived iPS cells and mES cells was established by qRT-PCR analysis conducted at different time points. The appearance of early CM markers, such as Brachyury, Mef2c, and Tbx5 (Figure 5e, upper panels), became evident earlier in CM-derived iPS cells than the other cell types. The expression of mature myocardial genes, such as $\alpha-M H C$ and $T N N I$, was higher in CM-derived iPS cells at the later stages of differentiation (Figure 5e, lower panels).

In addition, both cardiac-derived iPS lines could differentiate into three lineages found in the myocardium: CMs, ECs, and SMCs (Figure 6a). However, CM-derived iPS cells differentiated into CMs more often than into SMCs or ECs. In fact, the number of cells expressing $\alpha$-MHC and $\alpha$-SARC was significantly greater in those originating from $\mathrm{CM}$-derived iPS cells (Figure 6b).

$\mathrm{Ca}^{2+}$ response in $\mathrm{CM}$ - and CF-derived iPS cells after BMP2 exposure. Functional differences in commitment toward the cardiac phenotype was tested by comparing stimulus-induced $\mathrm{Ca}^{2+}$ release in differentiated $\mathrm{CM}$ - and CF-derived iPS cells and neonatal CMs, used as a reference (Figure 7). Responsiveness to caffeine, ${ }^{16}$ which acts on muscle-specific ryanodine receptor channels, was significantly higher in differentiated CM-derived iPS cells than in differentiated CF-derived iPS cells. Responsiveness to nicotine, acting on the nicotinic acetylcholine receptor (nAChR), was negligible in all cell types, and this ruled out differentiation toward skeletal muscle. Responsiveness to ATP and acetylcholine (ACh), mediated by inositol trisphosphate (IP3) release in many cell types and well represented in SMCs, ${ }^{17}$ was low in all cell types. The combined unresponsiveness to nicotine and infrequent response to IP3-mediated stimuli suggests that caffeine responsiveness was largely indicative of cardiac differentiation.

\section{Discussion}

In this report, we demonstrate that two myocardial cell types can be reprogrammed to generate iPS cells and that both can produce new cardiovascular cell populations in vitro, albeit with different efficiencies. In particular, we show for the first time that CMs are capable of generating iPS cells, and that when we used only OSK for the reprogramming of cardiac cells, the CM compartment, remarkably, was significantly more efficient in generating iPS cells than was the CF one. As post-natal CMs lose their proliferative capacity soon after birth, they possess a limited ability to survive and undergo cell replication in culture. In fact, CMs have a proliferation index that peaks at $\sim 10 \%$ on neonatal day 4.6 and declines rapidly to zero thereafter, ${ }^{18}$ and can only be forced to re-enter the cell cycle by transfecting cell-cycle regulatory genes or by exposure to growth factors. ${ }^{19}$ Therefore, it is conceivable that CM-derived iPS cells were formed from the residual number of $\mathrm{CMs}$ still actively cycling immediately after birth. Why CMs are better at producing iPS cells than CFs with the OSK protocol needs further investigation.

In addition, we show that CM-derived iPS cells are better at differentiating into functional CMs after exposure to BMP2 than are CF-derived iPS cells or mES cells, or MEF-derived iPS cells. In fact, CM-derived iPS cells generated more $\alpha-\mathrm{MHC}$ - and $\alpha$-SARC-positive cells and beating areas per dish after the cardiac differentiation procedure than did the other stem cell types. BMP2, a transforming growth factor $\beta$ (TGF- $\beta$ ) family member that is expressed in lateral endoderm and ectoderm, has an important role in cardiac development in vertebrate embryos. ${ }^{20}$ Expression of cardiac-specific proteins by TGF- $\beta$ is mediated by GATA4 and NKX2.5, ${ }^{21}$ and this factor was found to be upregulated more than threefold in CM-derived iPS cells compared with mES cells and CF-derived iPS cells when the threshold was set at a twofold change.

We also found that although CM- and CF-derived iPS cells are comparable to mES cells in the expression of transcription factors (e.g., endogenous Oct4) and surface markers for pluripotency (e.g., SSEA-1), they did show architecturespecific variations in their gene expression profiles, which may probably explain the differences in the yield of CMs following the induction of cardiogenic differentiation. Indeed, microarray analysis of global gene expression in iPS cells derived from both cardiac compartments identified upregulation of genes directly involved in cardiogenesis during the early days of embryonic life, such as Brachyury and fibroblast growth factor 2 (Fgf2). Brachyury facilitates BMP-induced differentiation of human and mouse pluripotent stem cells, and cooperates with Fgf2 (via ERK) in mesoderm rather than endoderm. ${ }^{22}$

Igfbp5, C1QTNF2, and Aldh3a1 were also found upregulated in iPS cells when the threshold was set at a twofold change. Igfbp5, found in the precardiac region and early cardiac crescent, ${ }^{23}$ encodes a member of the IGF-I-binding protein family that binds with high affinity to both IGF-I and IGFII. $^{24}$ Igfbp5 expression is induced by treatment with IGF-I in

Figure 2 Characterization of CM- and CF-derived iPS colonies. (a) Number of iPS colonies/35 mm-dish formed on MEFs after 15 days from transduction of CMs and CFs with OSK reprogramming factors. ${ }^{*} P<0.005$ : $t$-test $(N=5$ experiments for each clone). (b) Proliferation of post-natal CMs before and after transduction with OSK factors (left panel). BrdU labeling in a colony 15 days after sorting (right panel). Scale bar represents $200 \mu \mathrm{m}$. ${ }^{*} P<0.005$ : $t$-test ( $N=5$ experiments for each clone). (c) Morphology of CM- and CF-derived cells after 15 days from reprogramming (upper and lower left panels). Morphology of CM- and CF-derived iPS colonies at passages 2 and 20 (upper and lower right panels). Scale bars represent $200 \mu \mathrm{m}$. (d) Example of a 12-day-old CM-derived iPS clone stained for SSEA-1 (upper left panel); ALP expression in neonatal CM-derived iPS cells 15 days after transduction with OSK vectors (upper right panel); Oct4 and DAPI staining (lower right and left panels, respectively) in a colony formed from CMs. Scale bars represent $200 \mu \mathrm{m}$. (e) Teratoma formation 4 weeks after injection of CM-derived iPS cells in NOD-scid mice. Hematoxylin/eosin-stained sections demonstrating the presence of all three germ layers. (f) Normal karyotype of neonatal CM-derived iPS cells. (g) Two-week-old chimeric mouse, derived from a C57BL/6 blastocyst injected with CM-derived iPS cells obtained from a white CD-1 mouse, between two C57BL/6 wild-type littermates. CM-derived iPS cells are responsible for the agouti coat color. (h) qRT-PCR analysis of embryonic- and cardiac-marker genes in mES cells and CMs transduced with OSK factors ( $N=5$ experiments for each clone) 


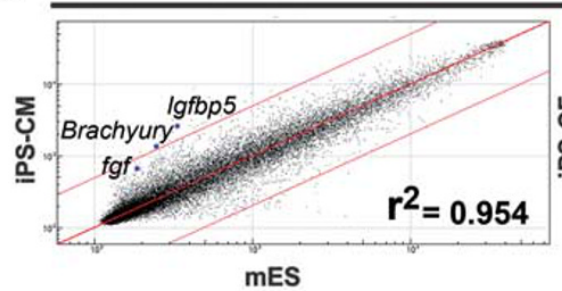

b

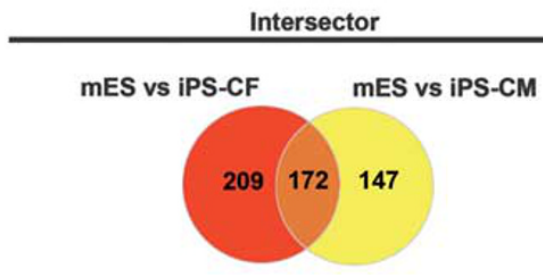

d

Gene Ontology
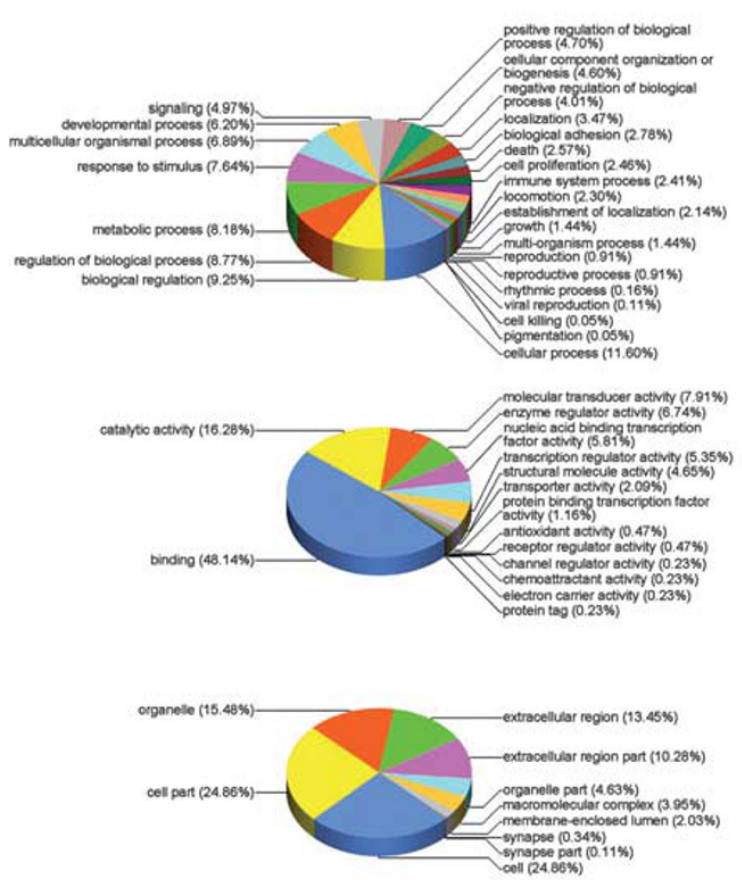

Correlation Of Global Gene Expression

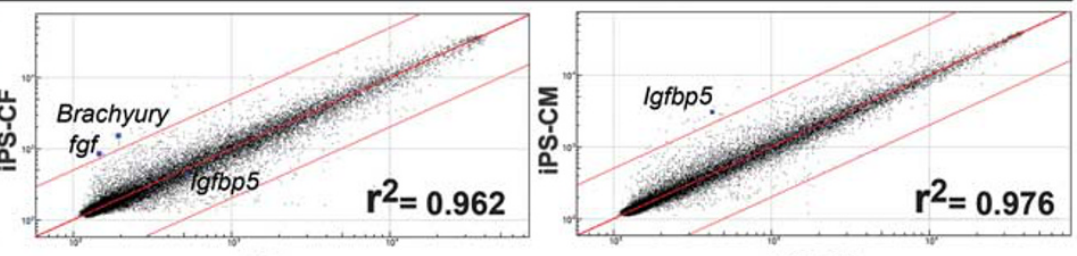

mES
Heat Map

c

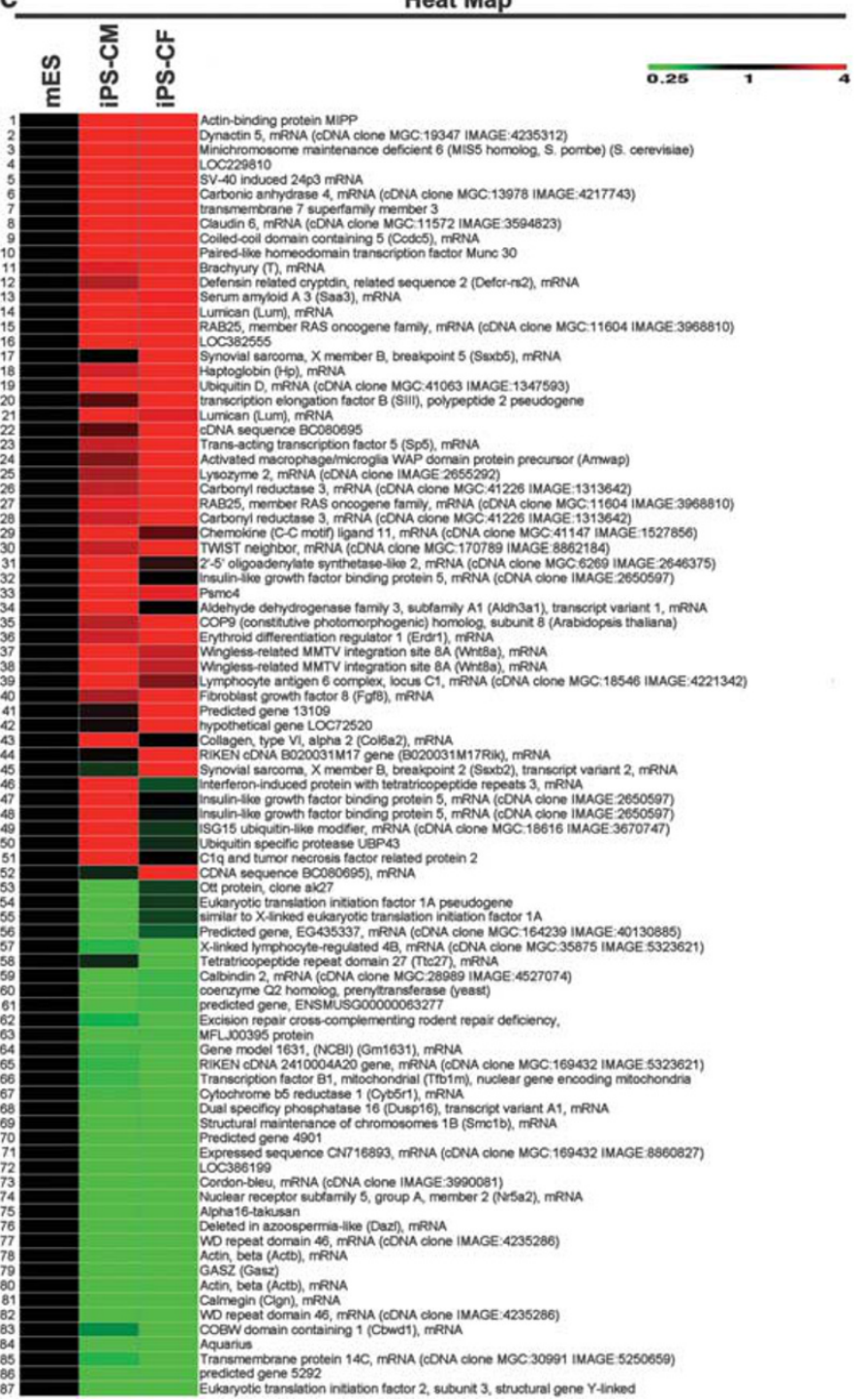

Figure 3 Genesifter analysis of changes in gene expression in $\mathrm{mES}$ cells and CM- and CF-derived iPS cells. (a) Comparisons of global gene expression in $\mathrm{mES}$ cells and CM-derived iPS cells (left panel), mES cells and CF-derived iPS cells (middle panel), and CM- and CF-derived iPS cells (right panel). (b) Venn diagram of the number of genes differentially expressed in CM- and CF-derived iPS cells and mES cells when a twofold change was taken as the threshold value. 381 genes were differently expressed between mES and CF-derived iPS cells, whereas 319 genes were differently expressed between mES and CM-derived iPS cells; 172 of these genes were differently expressed in both data sets ( $N=3$ experiments for each clone). (c) GeneSifter program heat map analysis of Illumina mouse gene array data from $\mathrm{mES}$ cells and $\mathrm{CM}$ - and CF-derived iPS cells: 87 genes were found to be significantly differentially expressed in CM- and CF-derived iPS cells compared with mES cells when a fivefold change was taken as the threshold value ( $N=3$ experiments for each clone). (d) $G O$ annotations of genes found differentially expressed at the twofold-change threshold. The domains are biological process (upper chart), cellular component (middle chart), and molecular function (lower chart) ( $N=3$ experiments for each clone)

both aortic and vascular SMCs in a wortmannin- and rapamycin-sensitive manner, suggesting that induction of Igfbp5 is downstream of the PI3K/mTOR signaling pathway. ${ }^{25}$
Higher expression of Igfbp5 may indicate different epigenetic imprinting in CM- and CF-derived iPS cells. Regarding C1QTNF2, it was reported that this protein induces the 
Differences In Gene Expression in Cardiac and Stemness Pathways

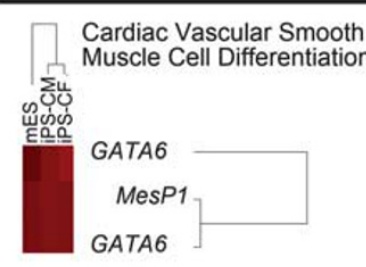

Cardiac Endothelial Cell Differentiation

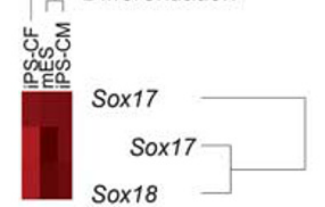

Cardiac Ventricle Formation Myocyte enhancer factor $2 \mathrm{C}$ \begin{tabular}{r|r|}
200 \\
150 \\
100 \\
50 \\
0
\end{tabular}$\prod_{\text {mES iPS-CM }} \prod_{\text {iPS-CF }}^{*}$

Cell Junction Organization

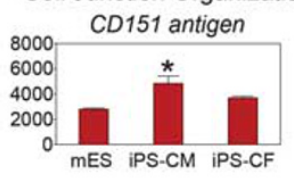

Cardiac Muscle Cells Differentiation

Acyl-Coenzyme A

dehydrogenase, medium chain$$
15000
$$

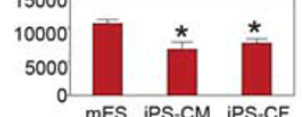

T-box 3

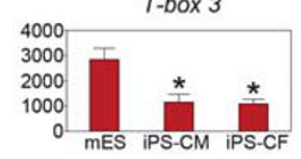

Stem Cell Development

Erythroid differentiation

regulator 1 (Erdr1)

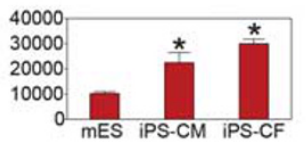

Wingless-related MMTV
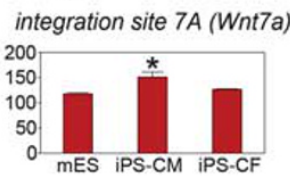

Kit oncogene (Kit), transcript variant 2

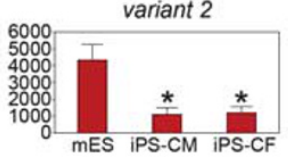

SRY-box containing gene 2 (Sox2)
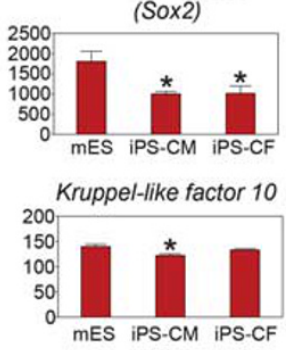

Cardiac Muscle Cells Development

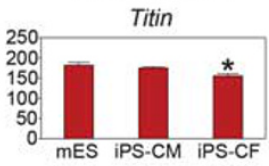

Cardiac Ventricle Development

MAD homolog 5 (Smad5)

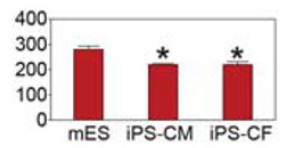

Cardiac Muscle Contraction

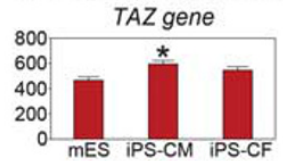

Embryonic Heart Tube Formation

Ovo-like 2

(Ovo/2), transcript variant $B$

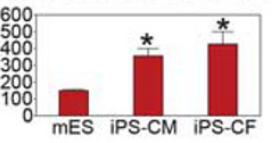

Low density lipoprotein receptor-related protein 6

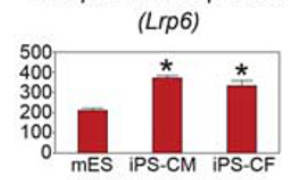

Hypoxia inducible factor 1 , alpha subunit

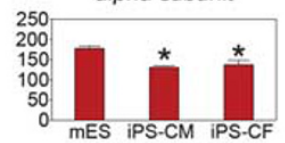

Catenin (cadherin associated protein), beta 1

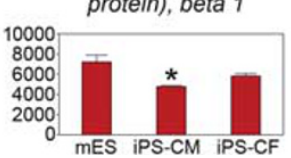

Figure 4 Cardiovascular and stemness pathways in CM- and CF-derived iPS cells. Heat maps (upper-left panels) and histograms of the genes found to be significantly up- or downregulated in different cardiovascular- and stem-cell-related pathways. The classification shown is that generated by GeneSifter. ${ }^{\star} P<0.05$ versus $m E S, A N O V A$ ( \pm S.E.M.). Gene expression by DNA microarray, analysis by GeneSifter software $(N=3$ experiments for each clone)

phosphorylation of AMPK in myocytes, resulting in increased glycogen accumulation and fatty acid oxidation. ${ }^{26}$ On the other hand, Aldh3a1 expression regulates proliferation, survival, as well as cellular response to oxidative stress. ${ }^{27}$ It is thus possible that this gene is implicated in CM differentiation.

Wnt7a is a highly upregulated gene in CM-derived iPS cells. Wnt7a mRNA expression is present in the ventricles of embryos, ${ }^{28}$ and Wnt and Wnt-related signal transduction contributes to mesodermal specification during early cardiogenesis and later cardiac development and maturation. ${ }^{29}$ It is well known that the formation of mesoderm is dependent on canonical Wnt signaling. ${ }^{30}$ In particular, Wnt/ $\beta$-catenin signaling uses Brachyury expression as a marker of activation. ${ }^{31}$ Thus, the upregulation of Wnt and Brachyury in CM-derived
iPS cells mimics the mechanism occurring in the early embryo for mesoderm induction, a prerequisite for the formation of cardiac progenitor cells. ${ }^{32}$

Another gene found highly upregulated in CM-derived iPS cells with respect to mES cells is Lrp6. This gene regulates specification, proliferation, and survival of cardiac progenitors and the expression of multiple cardiogenic genes, including Tcfap2a and Pax3, Is/1, Msx1/Msx2, Fgf8, and Mef2C during cardiogenesis. ${ }^{33}$

The final gene found significantly upregulated in CM-derived iPS cells is TAZ, which encodes for a WW domain-containing cofactor. TAZ co-associates with TBX5 and histone acetyltransferase proteins and mediates TBX5dependent gene activation during cardiac development. ${ }^{34}$ 
a

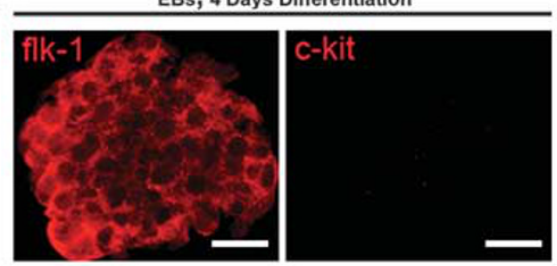

b Flk-1 Expression At Early Time Points
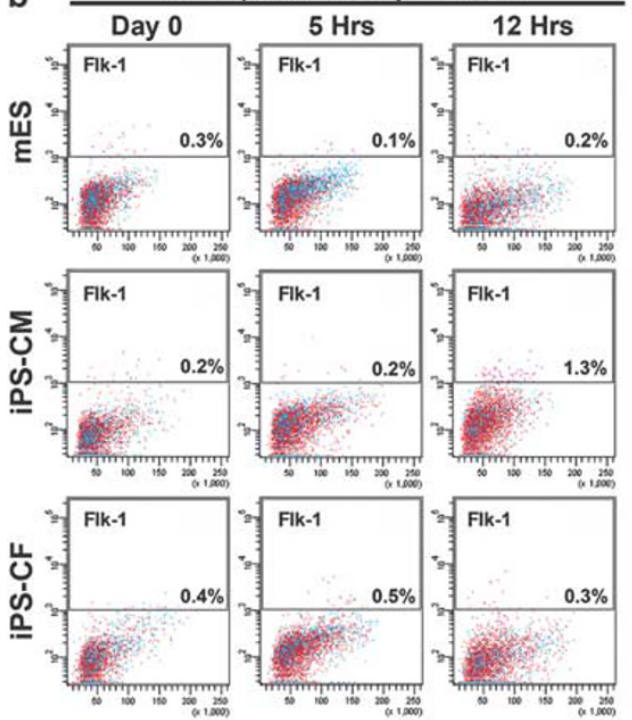

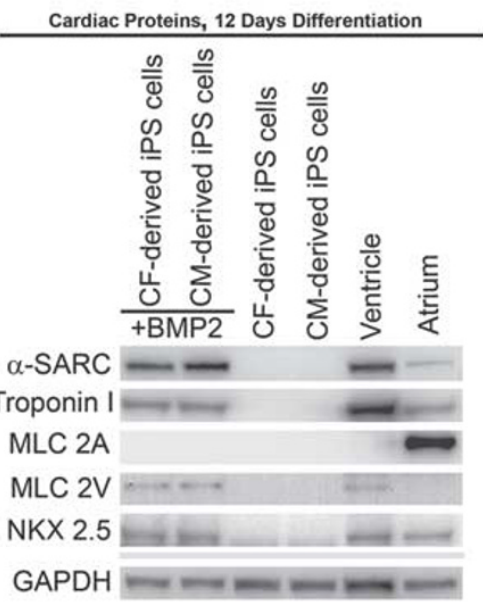

d

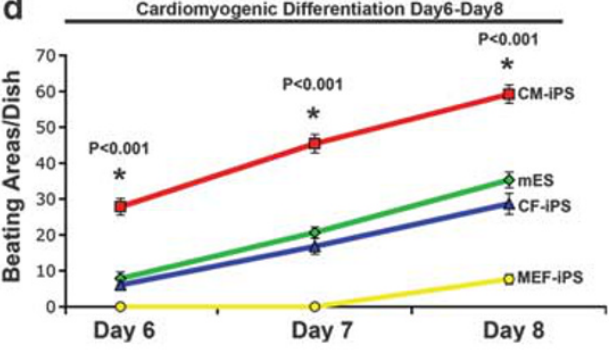

e

Dynamic Gene Expression During Differentiation
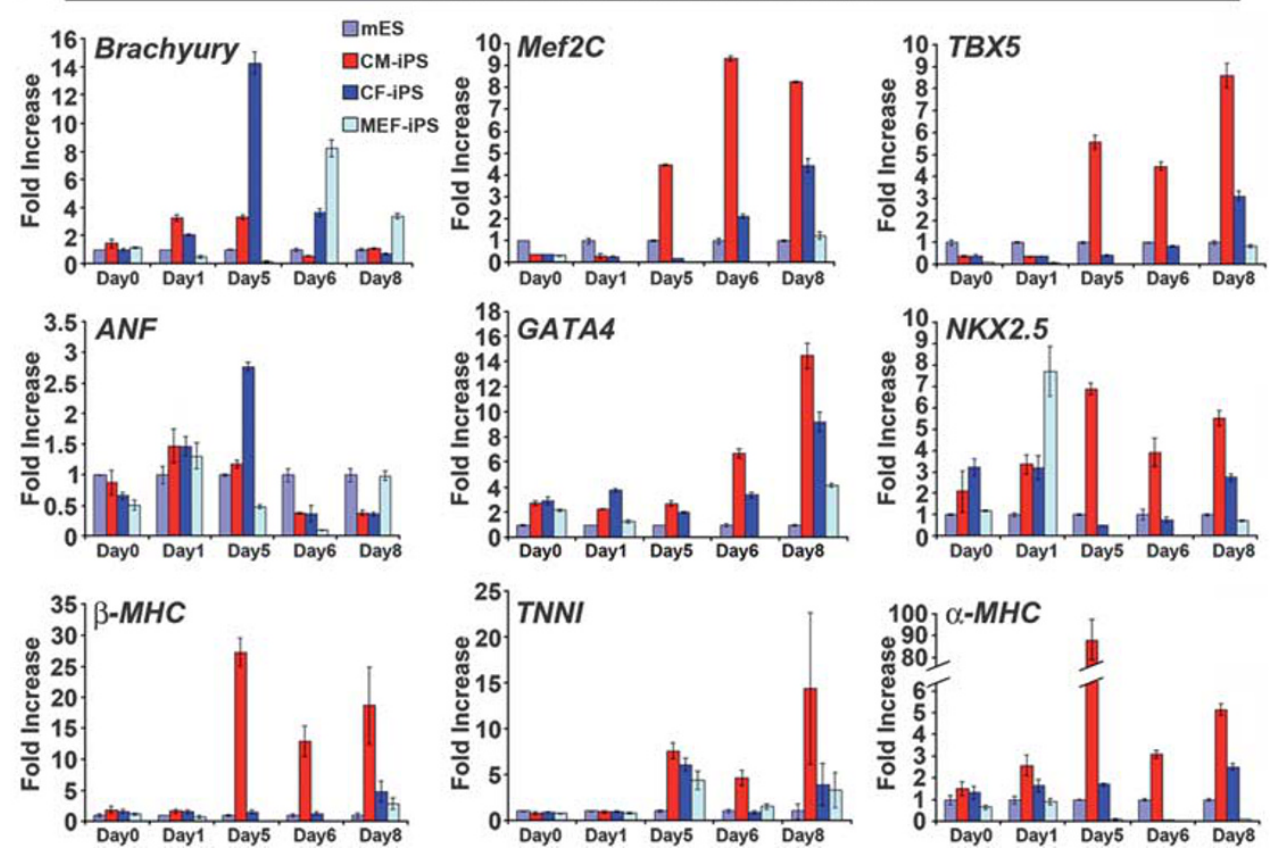

Figure 5 Cardiac lineage differentiation of CM- and CF-derived iPS cells in vitro. (a) Immunofluorescence for Flk-1 on an EB 4 days after exposure to BMP2, attesting in vitro differentiation into $\mathrm{CM}$ precursors (left panel). The lack of a signal for c-kit demonstrates the absence of putative cardiac progenitor cells that might have been responsible for the cardiogenic differentiation observed (right panel). Scale bars represent $100 \mu \mathrm{m}$. (b) Flow cytometry plots for Flk-1 expression at 0,5 , and $12 \mathrm{~h}$ after exposure to BMP2 in mES and CM- and CF-derived iPS cells. (c) Representative western blot of cardiac markers in differentiated and undifferentiated CF- and CM-derived iPS cells. Ventricular and atrial cells were used as positive controls. To remove uninformative areas, the panel has been cropped. (d) Number of beating areas in cultures derived from mES cells, CM- and CF-derived iPS cells, and MEF-iPS cells, 6,7 , and 8 days after exposure to BMP2. ${ }^{*} P<0.001$ : $t$-test ( $N=4$ experiments for each clone). (e) qRT-PCR analysis of early and late cardiac marker genes in mES cells and CM-, CF-, and MEF-derived iPS cells at different time points after transduction of cells with OSK transcription factors. An axis break has been introduced to remove uninformative areas ( $N=5$ experiments for each clone) 
TAZ also functions as a co-activator of GATA4, stimulating GATA4 activity on the ANF promoter in several cell lines. TAZ and GATA4 physically interact with each other thorough the N-terminal region of TAZ and activation domain of GATA4. Moreover, the Notch signaling pathway may regulate the activity of cardiac transcription factors, including GATA family proteins, by inhibiting TAZ. ${ }^{34}$

All these differences between CM-derived iPS cells and the other stem cell types investigated in our study strongly suggest that CM-derived iPS cells retain an 'epigenetic memory' of the cardiogenic fate of their originating cell type, which can influence the outcome of subsequent differentiation. On this point, it has been demonstrated that iPS cells derived from human fibroblasts, adipose tissue, and keratinocytes, and from granulocytes, muscle progenitors, fibroblasts, and lymphocytes ${ }^{35}$ show differences in gene expression that are indicative of transcriptional memory. The DNA methylation pattern of iPS clones has also been used to predict the somatic cell from which they were derived. $^{36}$

We have demonstrated as a proof of principle that CMs can generate iPS cells. We believe that these cells have significant investigative potential in the study of epigenetic mechanisms and the genes underlying cell cycling and cardiomyogenic differentiation processes.

\section{Materials and Methods}

Generation of lentiviral vectors. The lentiviral vector used to introduce a GFP transgene under the influence of a cardiac-specific promoter for the ultrapurification of CMs (TNNI3-GFP lentivirus vector), was produced as described elsewhere. ${ }^{11}$ See Supplemental Experimental Procedures.

The lentiviral vectors employed for the induction of reprogramming via OSK factors harbored bicistronic constructs expressing Oct4 and either Sox2 or KIf4 (OSK lentiviral vectors). These vectors, and those used for transducing all 4 Yamanaka factors (OSKM), were generated as described in Supplemental Experimental Procedures and Supplementary Table 1.

Myocardial cell cultures. CMs and CFs were obtained from of 1-day-old CD-1 mice (Charles River Laboratories, Wilmington, MA, USA) as previously described ${ }^{37}$ All animal procedures were performed in accordance with the Guide for the Care and Use of Laboratory Animals and approved by the Institutional Animal Care and Use Committee. CMs were seeded and kept overnight in medium containing BrdU to inhibit cell proliferation. See Supplemental Experimental Procedures.

Enrichment of the cardiomyocyte compartment. CMs and CFs were incubated with the TNNI3-GFP lentiviral vector 3 days after isolation. CMs were purified through cell sorting for GFP positivity $48 \mathrm{~h}$ after transduction. Sorted cells were plated on gelatin and cultured in CM expansion medium, ready for the reprogramming step.

Generation of iPS cells. To induce reprogramming, CMs and CFs were exposed 3 days after sorting to a mixture of equal volumes of the two OSK lentiviral vectors. Four days after transduction, cells were trypsinized and plated on a mouse MEF-feeder layer (Millipore, Billerica, MA, USA) at a density of $1.6 \times 10^{3} \mathrm{cells} / \mathrm{cm}^{2}$ and cultured in propagation medium composed of Knockout DMEM (Invitrogen, Carlsbad, CA, USA) containing 15\% KnockOut Serum Replacement (Invitrogen), $2 \mathrm{mM}$ L-glutamine, $100 \mu \mathrm{M}$ NEAA (non-essential amino acids), $10 \mathrm{ng} / \mathrm{ml} \mathrm{bFGF}$ (Peprotech, Rocky Hill, NJ, USA), $500 \mu$ M VPA (EMD Biosciences, San Diego, CA, USA), $100 \mu \mathrm{M} \beta$-mercaptoethanol (Invitrogen), $1000 \mathrm{U} / \mathrm{ml}$ LIF (Millipore), penicillin $(100 \mathrm{U} / \mathrm{ml})$, and streptomycin $(100 \mathrm{mg} / \mathrm{ml})$. Any iPS cells thus derived were maintained on MEFs in complete medium containing bFGF, VPA, and LIF (Supplementary Table 2).
Embryoid body formation and in vitro differentiation of iPS cells. Undifferentiated $\mathrm{CM}$ - and CF-derived iPS cells were cultured in propagation medium containing $2.5 \mathrm{ng} / \mathrm{ml}$. BMP2 (Invitrogen) for $12 \mathrm{~h}$. Cells were then harvested by trypsinization, spun down for $5 \mathrm{~min}$ at 1000 r.p.m., and resuspended in differentiation medium (DF medium) composed of DMEM, FBS, L-glutamine, NEAA, penicillin, and streptomycin. Successively, iPS cells were counted and diluted down to $25000 \mathrm{cells} / \mathrm{ml}$. The underside of bacterial dish lids was then spotted with 60 drops containing 500 cells each. After 4 days of hangingdrop culture, EBs were collected, seeded on gelatinized plates, and cultured in DF medium. EBs began to show areas of spontaneous contraction on day 6 . On the forth day, some EBs were collected, fixed in $4 \%$ paraformaldehyde, and stained for Flk-1; 10 days after BMP2 treatment, cells were fixed and stained for $\alpha$-SARC in order to identify any cells differentiated into CMs.

Chimera formation. iPS Cells deriving from CMs or CFs were aggregated with zona-pellucida-free eight-cell stage embryos to generate chimeras as described previously. ${ }^{38}$

Teratoma. formation. $5 \times 10^{5}$ cells/mouse were injected subcutaneously in five NOD-scid mice. Tumors were sought after 4 weeks.

Gene expression profiling. Expression of endogenous and exogenous genes was analyzed by RT-PCR (OSKM) and qRT-PCR (OSK) using standard protocols (Supplementary Tables 3 and 4). See Supplemental Experimental Procedures.

DNA microarray for whole-genome gene expression. BeadChip Array MouseWG-6 v2 (Illumina, San Diego, CA, USA) was used for whole-genome expression analysis following the protocol provided by Illumina. See Supplemental Experimental Procedures.

Western blotting analyses, immunohistochemistry and immunocytochemistry, and flow cytometry and cell sorting. These procedures were performed following standard protocols. See Supplemental Experimental Procedures.

$\mathrm{Ca}^{2+}$ response in CM- and CF-derived iPS cells after BMP2 exposure. Four days after BMP2 treatment, EBs were plated on glass coverslips and incubated in DF medium. During the incubation period, cells migrated from the EBs, forming an almost uniform cell layer. Freshly dissociated neonatal CMs were similarly plated and kept for 1 day in $\mathrm{CM}$ medium.

At day 7 , cells were loaded with the $\mathrm{Ca}^{2+}$-sensitive dye Fluo4-AM $(20 \mu \mathrm{M}, 1 \mathrm{~h}$ at $\left.37^{\circ} \mathrm{C}\right)$. Images $(512 \times 512$ pixels) were recorded (field size $441 \times 441 \mu \mathrm{m})$ with a confocal microscope (Leica TCS-SP2, Leica, Wetzlar, Germany) at a sampling rate of $1.2 \mathrm{~Hz}$. The ratio between dye fluorescence over time and its value in basal conditions $\left(F / F_{0}\right)$ was recorded. As cell boundaries were not visible in cell layers, response to the test intervention was quantified by the proportion of pixels corresponding to cell-covered areas ('cell pixels') in which $F / F_{0}$ changed more than a threshold value ('active pixels', Figure 7, right panels). The number 'cell pixels' was counted in digital images (Figure 7, left panels), obtained by applying a $F / F_{0}$ threshold to pixel values integrated over the recording period.

Cells were superfused with $\mathrm{Ca}^{2+}$-free $\left(0 \mathrm{mM} \mathrm{Ca}^{2+}+1 \mathrm{mM}\right.$ EGTA) Tyrode's solution. Substances ( $10 \mathrm{mM}$ caffeine, $200 \mu \mathrm{M}$ ATP, $30 \mu \mathrm{M} \mathrm{ACh}$, or $100 \mu \mathrm{M}$ nicotine), diluted in the superfusate, were applied by a fast switch perfusion system. Experiments were performed at $25 \pm 2{ }^{\circ} \mathrm{C}$

Statistics. All data are expressed as mean \pm S.E.M.. For cell studies, we analyzed a total of four different clones per cell type. In some instances, data were pooled from different cultures originating from each clone. Unpaired $t$-test or oneway ANOVA was used, as appropriate, to assess statistical significance between groups. A binomial distribution of values was assumed whenever ANOVA was applied to categorical data (SPSS 19); Bonferroni correction was applied to post-hoc comparisons. As the experimental unit is the cell and each cell is represented by multiple pixels, considering pixel numbers would unduly increase the analysis degrees of freedom; thus, to avoid overestimating significance, pixels were binned by using a bin size approaching cell size ( 200 pixels $\approx 150 \mu \mathrm{m}$ ). Pearson's correlation was used to test the internal correlation between the samples and between the groups involved in gene expression assay. No significant differences were found in the data obtained from each clone of a specific cell type. 
a

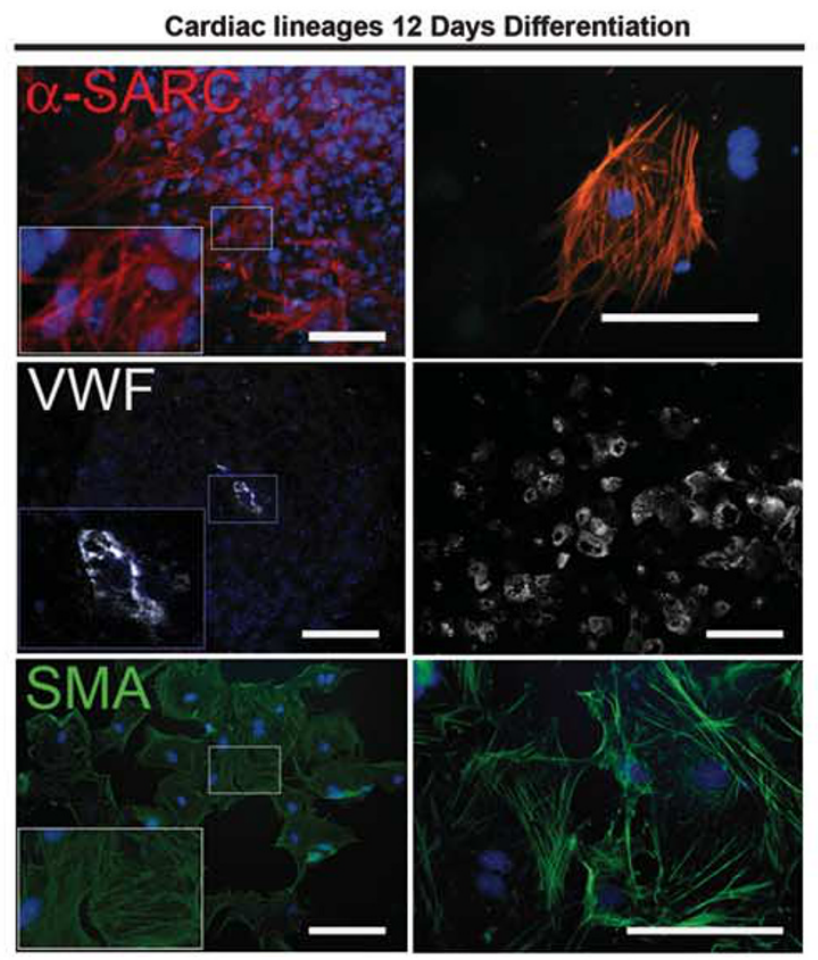

b

Cardiac Markers After 12 Days of Differentiation
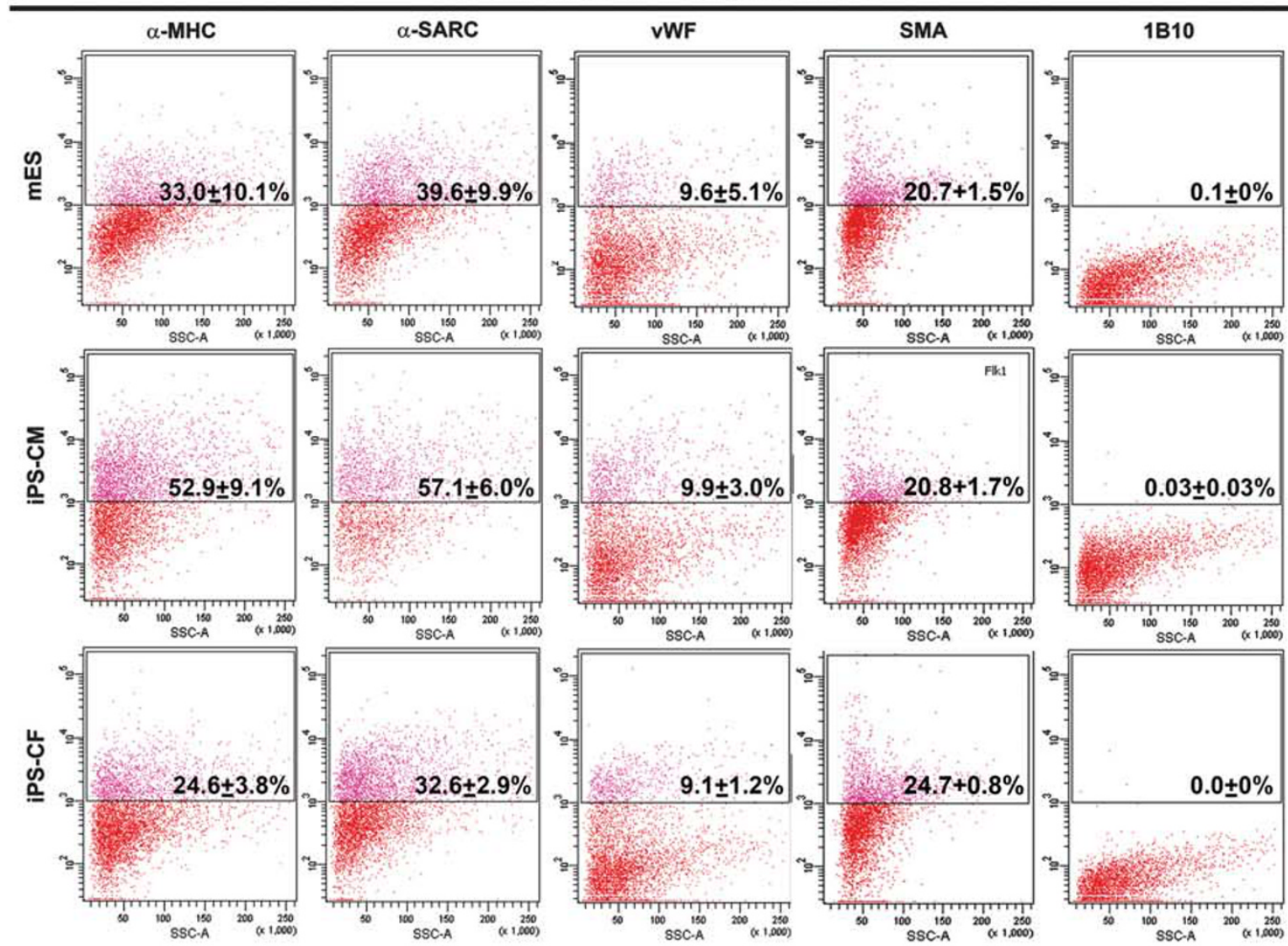
Figure 6 Quantitative evaluation of different cardiac lineages in vitro. (a) Representative fluorescence images for alpha-sarcomeric actin ( $\alpha$-SARC) (red), von Willebrand factor (VWF) (white), and smooth muscle actin (SMA) (green), demonstrating differentiation of CM-derived iPS cells toward CM, EC, and SMC lineages, 10 days after exposure to BMP2. Nuclei were stained with DAPI (blue). The areas in the rectangles are shown at higher magnification. Scale bars represent $40 \mu \mathrm{m}$. (b) Flow cytometry plots showing expression of $\alpha$-MHC (alpha-heavy myosin chain), $\alpha$-SARC, vWF, and SMA in mES cells and in CM- and CF-derived iPS cells, 10 days after BMP2 treatment ( $N=4$ experiments for each clone)

a

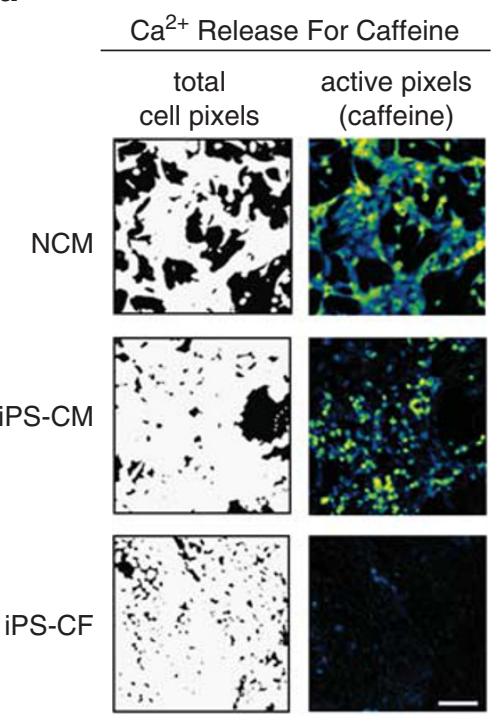

b

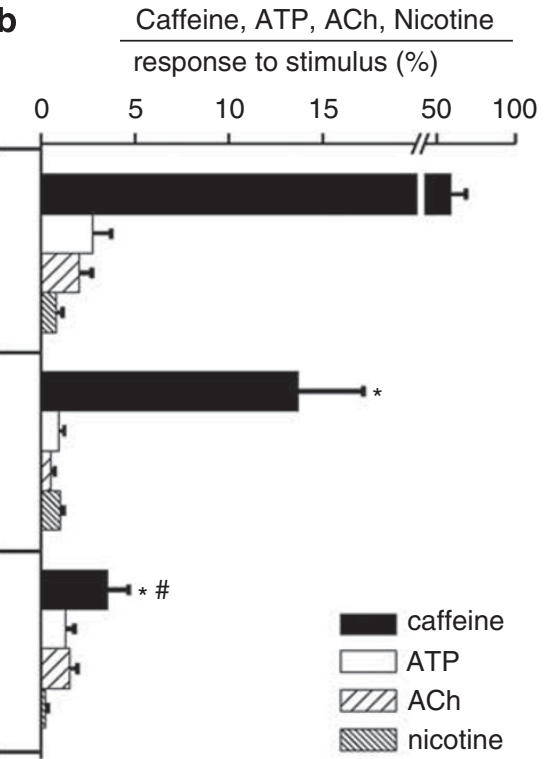

Figure 7 Responsiveness to $\mathrm{Ca}^{2+}$ releasing stimuli specific for muscle (caffeine, acting on ryanodine receptor (RyR), and nicotine, acting on nAChR) or shared by many cell types (ATP and Ach, acting through IP3). (a) Representative images of total cell pixels (in white, left panels) and caffeine activated pixels (F/F $F_{0}$ above threshold in color scale, right panels) for each experimental group. NCM, neonatal CM, used as a control. (b) Statistics of the proportion of 'cell pixels' activated by the test intervention in each experimental group (number of fields analyzed $=7,12$, and 10 for $\mathrm{CMs}$, differentiated CM- and CF-iPS cells, respectively). ${ }^{*} P<0.05$ versus $\mathrm{CMs}$; ${ }^{\#} P<0.05$ versus differentiated CM-derived iPS cells

\section{Conflict of Interest}

The authors declare no conflict of interest.

Acknowledgements. The backbone for OSKM lentiviral particles was kindly provided by Prof. AH Brivanlou (Rockefeller University, New York, NY, USA). The generation of chimeric mice was performed by Dr. lannicella and Dr. Azzolino (University of Torino, Torino, Italy). This work was supported by the Italian Ministry of Health: Ricerca Finalizzata 2007 (RF-INP-2007-652854); by the Super pig Program, cofinanced by Lombardy Region (Institutional Agreements no 14388A) and by the European Community - 7th Frame Program (NMP3-LA-2008-214402 and 294609CARDIOEPIGEN)

\section{Author contributions}

RR, GC, and CB designed research; RR, EDP, PP, RP, PC, CA, LS, AZ, EH, LN, and $\mathrm{CB}$ performed research; RR, GC and $\mathrm{CB}$ analyzed data; and RR, MVGL, GC and $\mathrm{CB}$ wrote the paper.

1. Murry CE, Lee RT. Development biology. Turnover after the fallout. Science 2009; 324 $47-48$

2. Krause $\mathrm{K}$, Schneider $\mathrm{C}$, Kuck KH, Jaquet $\mathrm{K}$. Stem cell therapy in cardiovascular disorders. Cardiovasc Ther 2010; 28: e101-e110.

3. Bearzi C, Leri A, Lo Monaco F, Rota M, Gonzalez A, Hosoda T et al. Identification of a coronary vascular progenitor cell in the human heart. Proc Natl Acad Sci USA 2009; 106 15885-15890.

4. Evans MJ, Kaufman MH. Establishment in culture of pluripotential cells from mouse embryos. Nature 1981; 292: 154-156.

5. Thomson JA, Itskovitz-Eldor J, Shapiro SS, Waknitz MA, Swiergiel JJ, Marshall VS et al. Embryonic stem cell lines derived from human blastocysts. Science 1998; 282: 1145-1147.
6. Wiedemann PM, Simon J, Schicktanz S, Tannert C. The future of stem-cell research in Germany. A Delphi study. EMBO Rep 2004; 5: 927-931.

7. Takahashi K, Yamanaka S. Induction of pluripotent stem cells from mouse embryonic and adult fibroblast cultures by defined factors. Cell 2006; 126: 663-676.

8. Takahashi K, Tanabe K, Ohnuki M, Narita M, Ichisaka T, Tomoda K et al. Induction of pluripotent stem cells from adult human fibroblasts by defined factors. Cell 2007; 131: 861-872

9. Yoshida Y, Yamanaka S. Recent stem cell advances: induced pluripotent stem cells for disease modeling and stem cell-based regeneration. Circulation 2010; 122: 80-87.

10. Vitale AM, Wolvetang E, Mackay-Sim A. Induced pluripotent stem cells: a new technology to study human diseases. Int J Biochem Cell Biol 2011; 43: 843-846.

11. Gallo $\mathrm{P}$, Grimaldi S, Latronico MV, Bonci D, Pagliuca A, Ausoni $S$ et al. A lentiviral vector with a short troponin-I promoter for tracking cardiomyocyte differentiation of human embryonic stem cells. Gene Ther 2008; 15: 161-170.

12. Theunissen TW, van Oosten AL, Castelo-Branco G, Hall J, Smith A, Silva JC. Nanog overcomes reprogramming barriers and induces pluripotency in minimal conditions. Curr Biol 2010; 21: 65-71.

13. Puceat M. Protocols for cardiac differentiation of embryonic stem cells. Methods 2008; 45 : 168-171.

14. Baba S, Heike T, Yoshimoto M, Umeda K, Doi H, Iwasa T et al. Flk1(+) cardiac stem/ progenitor cells derived from embryonic stem cells improve cardiac function in a dilated cardiomyopathy mouse model. Cardiovasc Res 2007; 76: 119-131.

15. Beltrami AP, Barlucchi L, Torella D, Baker M, Limana F, Chimenti S et al. Adult cardiac stem cells are multipotent and support myocardial regeneration. Cell 2003; 114: 763-776.

16. Altomare C, Barile L, Marangoni S, Rocchetti M, Alemanni M, Mostacciuolo G et al. Caffeine-induced $\mathrm{Ca}(2+)$ signaling as an index of cardiac progenitor cells differentiation. Basic Res Cardiol 2010; 105: 737-749.

17. Vermassen E, Parys JB, Mauger JP. Subcellular distribution of the inositol 1,4,5trisphosphate receptors: functional relevance and molecular determinants. Biol Cell 2004; 96: 3-17.

18. Soonpaa MH, Kim KK, Pajak L, Franklin M, Field LJ. Cardiomyocyte DNA synthesis and binucleation during murine development. Am J Physiol 1996; 271 (5 Part 2): H2183-H2189.

19. Pasumarthi KB, Field LJ. Cardiomyocyte cell cycle regulation. Circ Res 2002; 90: 1044-1054. 
20. Schultheiss $T M$, Burch JB, Lassar $A B$. A role for bone morphogenetic proteins in the induction of cardiac myogenesis. Genes Dev 1997; 11: 451-462.

21. Orkin SH. GATA-binding transcription factors in hematopoietic cells. Blood 1992; 80 575-581.

22. Bernardo AS, Faial T, Gardner L, Niakan KK, Ortmann D, Senner CE et al. BRACHYURY and CDX2 Mediate BMP-Induced Differentiation of Human and Mouse Pluripotent Stem Cells into Embryonic and Extraembryonic Lineages. Cell Stem Cell 2011; 9: 144-155.

23. Prall OW, Menon MK, Solloway MJ, Watanabe Y, Zaffran S, Bajolle F et al. An Nkx2-5/ Bmp2/Smad1 negative feedback loop controls heart progenitor specification and proliferation. Cell 2007; 128: 947-959.

24. Firth SM, Baxter RC. Cellular actions of the insulin-like growth factor binding proteins. Endocr Rev 2002; 23: 824-854.

25. Duan C, Liimatta MB, Bottum OL. Insulin-like growth factor (IGF)-I regulates IGF-binding protein-5 gene expression through the phosphatidylinositol 3-kinase, protein kinase B/Akt, and p70 S6 kinase signaling pathway. J Biol Chem 1999; 274: 37147-37153.

26. Wong GW, Wang J, Hug C, Tsao TS, Lodish HF. A family of Acrp30/adiponectin structural and functional paralogs. Proc Natl Acad Sci USA 2004; 101: 10302-10307.

27. Oraldi M, Saracino S, Maggiora M, Chiaravalloti A, Buemi C, Martinasso G et al. Importance of inverse correlation between ALDH3A1 and PPARgamma in tumor cells and tissue regeneration. Chem Biol Interact 2011; 191: 171-176.

28. Bond J, Sedmera D, Jourdan J, Zhang Y, Eisenberg CA, Eisenberg LM et al. Wnt11 and Wnt7a are up-regulated in association with differentiation of cardiac conduction cells in vitro and in vivo. Dev Dyn 2003; 227: 536-543.

29. Christiansen JH, Dennis CL, Wicking CA, Monkley SJ, Wilkinson DG, Wainwright BJ. Murine Wnt-11 and Wnt-12 have temporally and spatially restricted expression patterns during embryonic development. Mech Dev 1995; 51: 341-350.
30. Lindsley RC, Gill JG, Kyba M, Murphy TL, Murphy KM. Canonical Wnt signaling is required for development of embryonic stem cell-derived mesoderm. Development 2006; 133: 3787-3796.

31. Yamaguchi TP, Takada S, Yoshikawa Y, Wu N, McMahon AP. T (Brachyury) is a direct target of Wnt3a during paraxial mesoderm specification. Genes Dev 1999; 13: 3185-3190.

32. Gessert S, Kuhl M. The multiple phases and faces of wnt signaling during cardiac differentiation and development. Circ Res 2010; 107: 186-199.

33. Song L, Li Y, Wang K, Zhou CJ. Cardiac neural crest and outflow tract defects in Lrp6 mutant mice. Dev Dyn 2010; 239: 200-210.

34. Murakami M, Nakagawa M, Olson EN, Nakagawa O. A WW domain protein TAZ is a critical coactivator for TBX5, a transcription factor implicated in Holt-Oram syndrome. Proc Natl Acad Sci USA 2005; 102: 18034-18039.

35. Polo JM, Liu S, Figueroa ME, Kulalert W, Eminli S, Tan KY et al. Cell type of origin influences the molecular and functional properties of mouse induced pluripotent stem cells. Nat Biotechnol 2010; 28: 848-855.

36. Kim K, Doi A, Wen B, Ng K, Zhao R, Cahan $\mathrm{P}$ et al. Epigenetic memory in induced pluripotent stem cells. Nature 2010; 467: 285-290.

37. Condorelli G, Morisco C, Latronico MV, Claudio PP, Dent P, Tsichlis P et al. TNF-alpha signal transduction in rat neonatal cardiac myocytes: definition of pathways generating from the TNF-alpha receptor. FASEB J 2002; 16: 1732-1737.

38. Brancaccio M, Turco E, Hirsch E. Tissue-specific KO of ECM proteins. Methods Mol Biol 2009; 522: $15-50$

(c) This work is licensed under the Creative Commons Attribution-NonCommercial-No Derivative Works 3.0

Unported License. To view a copy of this license, visit http:/l creativecommons.org/licenses/by-nc-nd/3.0

Supplementary Information accompanies the paper on Cell Death and Differentiation website (http://www.nature.com/cdd) 